\title{
Editorial
}

\section{Knee surgery, arthroscopy, sports traumatology, cartilage repair and technology in orthopaedics: looking for a possible unifying concept for joint care}

The evolution of orthopaedic surgery over the past thirty years has, in much of the world, been strongly characterized by the concept of minimal invasiveness. This is a phenomenon that, thanks to the spread of arthroscopic techniques, has been particularly marked in the treatment of joint diseases. The knee was the first joint targeted by this revolutionary approach, followed by the shoulder and other large and medium sized joints. Today we are seeing an incredibly rapid increase in arthroscopic treatment of the hip joint.

A main advantage of the use of arthroscopy is that it has fostered a better awareness and a greater understanding of joint disease processes, and therefore made it possible to correlate clinical and pathological pictures. This, in turn, has promoted early diagnosis of conditions that can nowadays be treated conservatively, and also through programs designed to prevent evolution of the damage. Second, thanks to arthroscopy, we have been able to develop so-called anatomical surgical repair and reconstruction techniques, designed to restore the joint anatomy to how it was before the onset of the lesion. This seemingly simple concept has had a revolutionary impact; over the years, it has increasingly taken the place of so-called functional surgery - a therapeutic approach whose primary aim was to eliminate the main symptom, even if this meant creating a "new anatomy", which in the long term, however, often proved detrimental to joint, altering its kinematics and allowing progression of the arthritic damage.

At the same time, technological and basic scientific research has fostered a growing evolution and application of biotechnologies and regenerative medicine in the repair and reconstruction of connective tissue, primarily joint cartilage. Replacement surgery, too, has kept up with the pace of change, using innovative materials, less and less invasive and more functional prostheses, and sophisticated technologies that are increasing the precision and reproducibility of procedures, and improving the tolerability and survival of implants.

A particularly important field of application for cutting-edge regenerative and minimally invasive methods is the care of active young patients, athletes in particular. Sports traumatology is a field that has always needed particularly effective therapeutic approaches, in terms of the speed of healing and the reliability of the result, given that athletes are patients with high functional demands, who are also more exposed to the risk of relapses due to traumatic injury or overuse.

Over time, as a result of these developments, the scientific community has been engaged in a sort of ongoing process of aggregation and disaggregation in relation to the above mentioned areas of interest, sometimes creating societies interested in a range of topics (e.g. ESSKA and ISAKOS), and sometimes preferring to create separate societies dealing with specific subspecialties, which may be related to a particular anatomical area (knee surgery, shoulder surgery, etc.), surgical technique (arthroscopy, innovative arthroscopy, etc.), or field of application (sports traumatology, cartilage treatment, etc.). At both national and international level, this process has been reflected in the creation, splitting and grouping of scientific journals and societies; meanwhile, other societies have, in competition, set out to cover the same topics. Obviously, this situation has resulted in an overlapping of programs and projects, and thus a deeply regrettable waste of time and resources. All this can perhaps be explained by the fact that we have not yet managed to form a clear idea of scientific societies (and magazines) that, although specialized, remain "above" the "subspecialty" level. The question that needs to be asked is what do all the above-mentioned experiences have in common? The answer is easy for those able to see beyond the details: the treatment of joint diseases.

By adopting a broad perspective, it is possible to eliminate the conflict between the subspecialties, which nevertheless retain their identity through their target of reference, and the "super-specialty", whose role is to collect the available experience and transfer it to the end user, who may be a congress participant or journal reader.

Accordingly, Joints aims to be a journal that focuses on the care of joints generally. In this issue, for example, we bring together articles covering surgery of the hip (1,2), knee (3), shoulder (4), elbow (5) and ankle (6), as well as replacement surgery $(1,2)$, arthroscopic surgery (5, 7) and open surgery $(3,6)$, and cartilage disease $(7,8)$, regenerative medicine $(7,9)$ and sports traumatology $(10)$. All these are areas of interest that belong to our scientific world, and you find them all here in the same issue-together.

References

1. Graceffa A, Indelli PF, Latella L, et al. Clinical outcome of design modifications to the CLS Spotorno Stem in total hip replacement. Joints. 2016;4(3):134-141.

2. Grano GF, Pavlidou M, Todesco A, et al. A new anterolateral surgical approach for total hip replacement. Joints. 2016;4(3):148-152.

3. Cottino U, Deledda D, Rosso F, et al. Chronic knee extensor mechanism lesions in total knee arthroplasty: a literature review. Joints. 2016;4(3):159-164.

4. Gigante $A$, Bottegoni $C$, Milano $G$, et al. Semitendinosus and gracilis free muscle-ten- don graft for repair of massive rotator cuff tears: surgical technique. Joints. 2016;4(3): 189-192.

5. Arrigoni $P, D^{\prime}$ Ambrosi $R$, Cucchi $D$, et al. Arthroscopic fixation of coronoid process fractures through coronoid tunnelling and capsular plication. Joints. 2016;4(3):153-158.

6. Ortolani A, Bevoni R, Russo A, et al. Posterior tibial tendon displacement behind the tibia and its interposition in an irreducible isolated ankle dislocation: a case report and literature review. Joints. 2016;4(3):183-188.

7. Mancò A, Goderecci R, Rughetti A, et al. Microfracture versus microfracture and platelet-rich plasma: arthroscopic treatment of knee Ghondral lesions. A two-year follow-up study. Joints. 2016;4(3):142-147.

8. Tarabella V, Filardo G, Di Matteo B, et al. From loose body to osteochondritis dissecans. A historical account of disease definition. Joints. 2016;4(3):165-170.

9. Saccomanno MF, Capasso L, Fresta L, Milano G. Biological enhancement of graft-tunnel healing in anterior cruciate ligament reconstruction. Joints.2016;4(3):174-182.

10. North AS. Rockwood grade I and II acromioclavicular injuries: as benign as commonly believed? Joints. 2016;4(3):171-173. 